\title{
Changes in the composition of the hamster zona pellucida after fertilization in vivo but not in vitro
}

\author{
C. R. Brown, N. Clarke, M. Aiken* and B. D. Bavister* \\ Institute of Animal Physiology and Genetics Research, Babraham Hall, Cambridge CB2 4AT, UK; \\ and ${ }^{*}$ Department of Veterinary Science, University of Wisconsin, Madison, WI 53706, USA
}

\begin{abstract}
Summary. Hamster zonae pellucidae were obtained from follicular oocytes, superovulated eggs, and eggs fertilized in vivo or in vitro. Zonae were labelled with $N$-succinimidyl-3(4-hydroxy, 5-[ ${ }^{125}$ I]iodophenyl)propionate, and compared on singleand two-dimensional SDS-PAGE. Single-dimensional electrophoresis showed considerable differences between zona categories in the amount of label that they incorporated; follicular zonae incorporated the least label and zonae from eggs fertilized in vivo the most. On two-dimensional electrophoresis, polypeptides from 3 of the 4 zona categories migrated into 4 major groups: two of these groups each with $M_{\mathrm{r}} 150000-250000$ were within the $M_{\mathrm{r}}$ range of $Z \mathrm{P} 1$, and two others, at $M_{\mathrm{r}} 90000$ and 55000 , appeared to be analogous to ZP2 and ZP3, respectively. The fourth zona category (zonae from eggs fertilized in vivo) showed a changed polypeptide profile as well as incorporating the most label; one of the polypeptides, $M_{\mathrm{r}} 150000-250000$, was undetectable, but a train of $M_{\mathrm{r}} 70000-90000$ polypeptides and a discrete polypeptide at $M_{\mathrm{r}} 20000$ were new. Since this changed profile did not occur in zonae from superovulated eggs, or in zonae from eggs fertilized in vitro, a synergism between oviducal factors and factors from the spermatozoon or egg, or both, towards the zona in vivo is indicated.
\end{abstract}

Keywords: fertilization; zona pellucida; electrophoresis; hamster

\section{Introduction}

An essential stage in the process of mammalian fertilization is the passage of the spermatozoon through the zona pellucida, the relatively thick glycoprotein matrix which encompasses the eggcell. It is evident that the spermatozoon requires pre-fertilization 'conditioning' not only brought about by the oviducal environment but also by interaction with the zona pellucida before it is able to complete its path to the egg membrane. Recent investigations have highlighted the uniqueness of this particular egg component and its possible, likely and known functions are very well considered in a recent compilation by Dietl (1988). Our most advanced knowledge of the composition, structure and function of the mammalian zona pellucida has been derived from studies on the mouse and pig but information has been steadily accruing from others. So far it seems fairly general that the zona pellucida is composed of three inherent proteins ZP1, ZP2 and ZP3, each richly glycosylated and all of which exhibit charge heterogeneity when subjected to two-dimensional gel electrophoresis. Wassarman et al. (1988) have identified and detailed a critical role for ZP3 $\left(M_{\mathrm{r}} 83000\right)$ in the mouse as the zona moiety with sperm receptor properties. A similar role has been assigned to a zona pellucida protein of $M_{\mathrm{r}} \sim 58000$ in the pig (Sacco et al., 1984, 1989). However, it has become evident that the composition of the zona pellucida may become modified after the oocyte is ovulated. For several species it has been shown that glycoproteins secreted from epithelial cells of the oviduct bind firmly and quite specifically to the zona pellucida of the newly ovulated egg (Brown \& Cheng, 1986; Lévéillé et al., 1987; Oikawa et al., 1988; Gandolfi et al., 1989; Boatman, 
1990). This possibly indicates a more extensive role for the zona pellucida than originally considered, and merits further investigation.

To be able to understand the overall role of the zona pellucida it is inevitable that we must first study the compositional changes that occur from the time the oocyte matures within the follicle to the time that the zona releases the preimplantation embryo. The number of species in which a significant profile of developmental changes has been attained is very limited. A good model for the study of the zona is the hamster, a species in which recovery of embryos fertilized in vivo has for some time been possible and in which a repeatable method for fertilization in vitro has now been defined (Bavister, 1989). Since a sensitive means of zona analysis also exists, we decided to look for changes in the composition of the hamster zona at three stages in its development in vivo, i.e. follicular, oviducal, and fertilized, and to compare these zona proteins with those of zonae recovered from embryos produced by in-vitro fertilization.

\section{Materials and Methods}

Chemicals and media. All general chemicals were of the purest grade available. Chemicals for electrophoresis were obtained from Sigma Chemical Co. (London, UK) or from BDH (Poole, Dorset, UK). The reagent $N$-succinimidyl 3-(4)hydroxy, 5-[125I]iodophenyl)-propionate (Bolton \& Hunter, 1973) and ${ }^{14} \mathrm{C}$-labelled molecular weight markers were from Amersham International, Bucks, UK. The composition of media used for the collection of eggs and fertilization in vitro were as detailed by Bavister (1989). Collection, preparation and co-incubation of gametes specifically for fertilization in vitro were carried out in TALP-PVA. This is a modified Tyrode's solution containing bovine serum albumin ( $3 \mathrm{mg} / \mathrm{ml}$ ) originally designated TALP (Bavister \& Yanagimachi, 1977) but now further modified by inclusion of $1 \mathrm{mg}$ polyvinylalcohol/ml (PVA). Subsequent manipulation of embryos was carried out in TALP-PVA without albumin (TL-PVA) as were the collection and preparation of follicular oocytes and superovulated eggs. EGTA (2 mM) was included in TL-PVA.

Collection of follicular oocytes. Golden hamsters 2-3 months old were injected with 20 i.u. PMSG (Pregnyl, Organon [from Diosynth, Chicago, IL, USA]) at $09: 00 \mathrm{~h}$ on the morning of the post-oestrous discharge (=Day 1) followed by 20 i.u. hCG (Sigma Chemical Co.) during the evening of Day 3. After $10 \mathrm{~h}$ they were killed and their ovaries excised and immersed in Medium TL-PVA. Stimulated follicles were aspirated using a 1 -ml syringe fitted with a 26-gauge needle and oocytes, surrounded by corona radiata, were transferred to fresh medium. Corona radiata was removed using a series of hand-pulled glass pipettes with increasingly fine bores.

Collection of superovulated eggs. Animals received hormone treatment as above but were killed $16 \mathrm{~h}$ after hCG. Oviducts were flushed with Medium TL-PVA to yield approximately 50 eggs per animal. Cumulus oophorus and corona radiata were removed mechanically as described above.

Collection of eggs fertilized in vivo. Animals received PMSG as described above and were mated during the evening of Day 4. One-cell embryos were collected in Medium TL-PVA at 12:00 h on Day 5 and were relatively free of cumulus and corona radiata; remnants were easily removed with a fine-bore pipette, as were any spermatozoa bound to the zona pellucida.

Collection of eggs fertilized in vitro. Females were superovulated as described above and eggs in cumulus were recovered and maintained in Medium TALP-PVA. Epididymal spermatozoa were collected from mature males and incubated in medium for $4 \mathrm{~h}$ at $2 \times 10^{6}$ cells $/ \mathrm{ml}$. Just before $4 \mathrm{~h}$, superovulated eggs were gathered and at $4 \mathrm{~h}$ were incubated with spermatozoa diluted to $2 \times 10^{5}$ cells $/ \mathrm{ml}$. After $15-20 \mathrm{~min}$ when eggs were virtually denuded of cumulus and corona radiata they were transferred to a fresh batch of spermatozoa at the same concentration. After a further $2 \mathrm{~h}$ spermatozoa bound to the zona pellucida were removed by pipetting and embryos were transferred to Medium TL-PVA (through 3 washes) for processing of zonae.

Removal of zonae pellucidae. The zona pellucida was fractured with a pipette with a bore of slightly smaller diameter than the zona, and broken vitelli were released rapidly into Medium TL-PVA which contained $0.5 \mathrm{~mm}$ phenylmethylsulphonylfluoride (PMSF). Zonae were removed in groups of 5 and then placed into fresh medium without PMSF. When enough zonae had been gathered they were ${ }^{125} \mathrm{I}$-labelled without delay as described below.

${ }^{125}$ I-labelling of zonae pellucidae. This was carried out essentially as described by Brown \& Cheng (1985). Groups of 50 zonae in $10 \mu \mathrm{l}$ Medium TL-PVA received $2 \cdot 5 \mu \mathrm{Ci}$ of the reagent $N$-succinimidyl 3-(4-hydroxy, 5 -[ $\left[{ }^{125} I\right]$ iodophenyl) propionate in $20 \mu 10 \cdot 1 \mathrm{M}$-borate buffer $\mathrm{pH} 8.5$ (Bolton \& Hunter, 1973). After $15 \mathrm{~min}$ at $25^{\circ} \mathrm{C}$ the zonae were transferred through 3 dishes of medium and 30 zonae of each category (follicular, oviducal, fertilized in vitro and fertilized in vivo) were dissolved in the appropriate electrophoresis buffer.

Electrophoresis. Single-dimensional SDS-polyacrylamide gel electrophoresis was carried out under reducing and non-reducing conditions utilizing 5-20\% (w/v) polyacrylamide gradient slab gels $80 \mathrm{~mm} \times 10 \mathrm{~mm} \times 0.5 \mathrm{~mm}$ containing $0.1 \% \mathrm{w} / \mathrm{v}$ SDS. Zonae were denatured in sample buffer consisting of $62.5 \mathrm{~mm}-\mathrm{Tris}-\mathrm{HCl} \mathrm{pH} 8.6,2 \%(\mathrm{w} / \mathrm{v})$ SDS 
and $10 \%(\mathrm{w} / \mathrm{v})$ glycerol; $5 \%(\mathrm{w} / \mathrm{v}) 2$-mercaptoethanol was included when reduction of the sample was required. Five zonae containing up to 50000 c.p.m.(total) ${ }^{125} \mathrm{I}$ were applied to each track. For two-dimensional electrophoresis the method of O'Farrell (1975) was used; proteins from 15 zonae were denatured and reduced in lysis buffer containing urea and 2-mercaptoethanol, and were then focussed by 7260 volt hours on rod gels containing mixed ampholytes. Included in $10 \mathrm{ml}$ gel-mix were $0.60 \mathrm{ml}$ of $\mathrm{pH} \mathrm{5-7}$ and $0.115 \mathrm{ml}$ of $\mathrm{pH} \mathrm{3-10} \mathrm{ampholytes} \mathrm{(Pharmacia} \mathrm{Ltd,} \mathrm{Milton}$ Keynes, Bucks, UK). The second dimension was run on a polyacrylamide gradient of $8-15 \%(\mathrm{w} / \mathrm{v}) .{ }^{125} \mathrm{I}$-labelled proteins were detected by autoradiography on Kodak X-Omat film, and the densitometric scans were carried out with a Joyce-Loebl Chromoscan 3 densitometer.

\section{Results}

\section{Single-dimensional SDS-PAGE}

On non-reducing and reducing gels there were differences between the 4 categories of zonae. The major differences in labelling of zona polypeptides were between zonae that had not been exposed to spermatozoa and those that had, the difference being more in the intensity of labelling rather than in the variety of polypeptides labelled (Fig. 1). Follicular zonae incorporated the least amount of label, and superovulated zonae slightly more than follicular zonae; a further increase in incorporation was evident in zonae from superovulated eggs fertilized in vitro but by far the most label was taken up by zonae of superovulated eggs fertilized in vivo. On non-reducing gels follicular zonae (i) labelled diffusely over a range of $M_{\mathrm{r}} \sim 150000-250000$ within which was a slightly heavier labelled area of $M_{\mathrm{r}} \sim 200000$; also, a more discrete section of label was present at $M_{\mathrm{r}} 50000-55000$. Non-reduced zonae from superovulated eggs (ii) gave much the same pattern but with more intense labelling in the higher molecular weight area and faint but discrete labelling of a polypeptide at $M_{\mathrm{r}} 70000$. The most striking difference was seen in zonae from eggs fertilized in vivo in that there was a several-fold increase in the intensity of labelling of the material at $M_{\mathrm{r}} 180000-240000$ (iii). Zonae from eggs incubated with spermatozoa in vitro (iv) also showed an increase of labelling at $M_{\mathrm{r}} 180000-240000$ but in this case not so marked. There was also increased labelling at $M_{\mathrm{r}} 50000-55000$ in both categories of fertilized egg.

When the reduced samples were analysed follicular zonae showed much the same diffuse labelling as when non-reduced (i). Reduced superovulated zonae (ii) gave a marginally different profile from non-reduced in that there appeared to be less label at the $M_{\mathrm{r}} 200000$ area of the gel. Zonae from eggs fertilized in vivo showed marked intensification of label at $M_{\mathrm{r}} 180000-220000$ with a narrowing of the band (iii). Appearing also was an additional area of label at $M_{\mathrm{r}} 90000-125000$. Zonae from eggs fertilized in vitro (iv) showed much less labelled material at $M_{\mathrm{r}} \sim 200000$ than did eggs fertilized in vivo but differences between the companion gels were seen. The left hand gel showed a labelled area $M_{\mathrm{r}} 90000-120000$ which the right hand gel did not, but there was more label at $M_{\mathrm{r}} 180000-250000$ in the latter.

\section{Two-dimensional SDS-PAGE}

Under reducing conditions follicular zonae gave a profile similar to those from ovulated eggs (Fig. 2). An area of label occupied a molecular weight range of $M_{\mathrm{r}} \sim 150000-250000$ with an isoelectric point range of $\sim 5 \cdot 5-6.8$ (a), within which were 4-6 barely discernible heavier labelled areas. A less diffuse area of label (b) covered a similar molecular weight range to (a) but possessed an isoelectric point range averaging $\sim 4 \cdot 8$. Below (b) at approximately $M_{\mathrm{r}} 90000$ there was an area of label (c) which varied more in its density than (b) but which focussed to a similar isoelectric point. Isolated at $M_{\mathrm{r}} 55000$ was a slightly more acid polypeptide focussing at a point approximately $4.5(\mathrm{~d})$. Also showing on the gel of follicular and ovulated zonae were several much smaller spots of label ranging from approximately $M_{\mathrm{r}} 90000$ to 30000 across a range of isoelectric point values. Zonae from eggs fertilized in vitro gave much the same profile except that many of the smaller areas of label were no longer present. Zonae of eggs from the in-vivo fertilization procedure gave quite a different profile. As was evident from the reduced one-dimensional gel the higher 


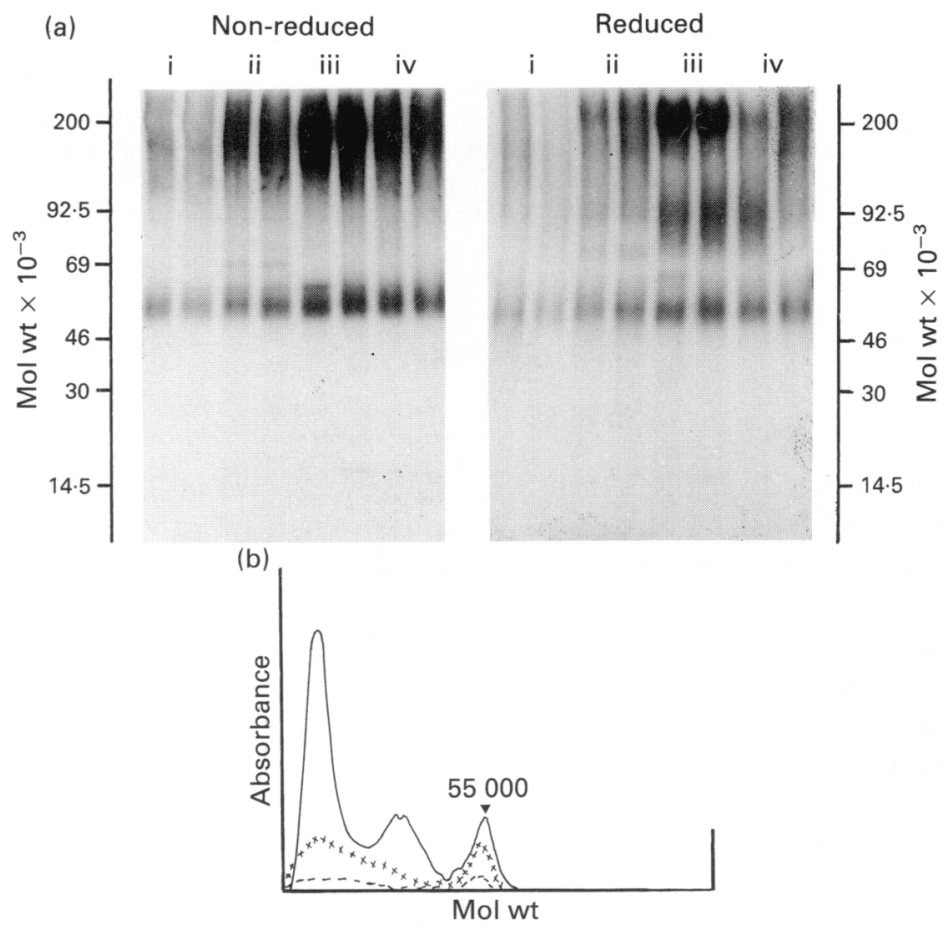

Fig. 1. Single-dimensional SDS-PAGE analysis of hamster zonae pellucidae. (a) Separation of polypeptides from follicular oocytes (i), superovulated eggs (ii), eggs fertilized in vivo (iii) and eggs fertilized in vitro (iv). Two samples of each category are shown, each containing 5 labelled zonae. (b) Electrophoretogram of zona polypeptides separated under reducing conditions as shown in (a). Follicular zonae (i) ---, superovulated zonae (ii) $-\mathbf{x}-\mathbf{x}-$ and zonae from eggs fertilized in vivo (iii) —.

molecular weight area of $M_{\mathrm{r}} 150000-250000$ lost its lower molecular weight component and appeared as a tighter area of $M_{\mathrm{r}} 190000-250000$. The polypeptide (b) was largely if not completely absent, whereas there was a train of possibly 7 polypeptides (e) with $M_{\mathrm{r}}$ values ranging from approximately 70000 to 90000 and isoelectric points from approximately 5 to 6 . The polypeptide $M_{\mathrm{r}} 50000-55000$ (d) showed no change, but a discrete and heavily labelled polypeptide $M_{\mathrm{r}} 20000$ was evident (f) which did not appear (except, possibly, for a small trace) on the single-dimensional gels.

\section{Discussion}

Compared with some other species, especially the mouse and pig, relatively little work has been carried out on the composition and structure of the hamster zona pellucida. In terms of the electrophoretic analysis of the hamster zona pellucida, reports on the number of major components and their respective molecular weights have varied, presumably for preparational and analytical reasons rather than for inherent compositional reasons. Gwatkin (1978) reported 3 components with molecular weights of 55000, 80000 and 100000 , Ahuja \& Bowell (1983) also reported 3 of molecular weight 80000,150000 and 240000 and Oikawa et al. (1988) reported 4 components of 55-70 000, 110-130000, 145-170000 and 200-240000 of which the largest was considered to be of oviducal origin. Oikawa et al. (1988) have assigned current nomenclature linked to an order of ascending molecular weight to the four zona components that they have identified, ZP3, ZP2, 


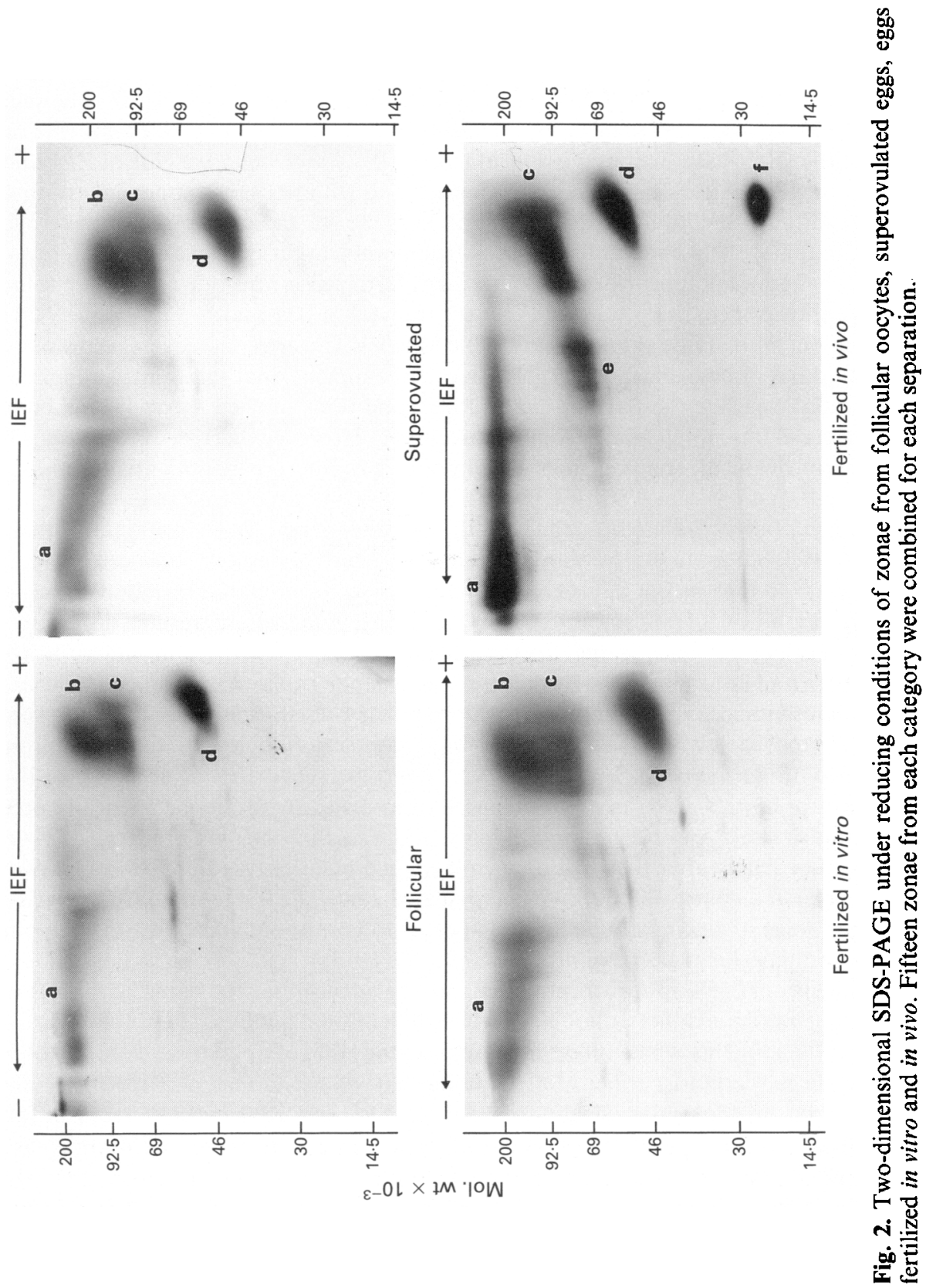


ZPI and ZP0. We too have seen 4 major components in follicular and ovulated zonae but also a modified composition in zonae that have been exposed to spermatozoa in vivo but not in vitro.

For ease of discussion we refer to the zona components alphabetically. The 4 components that we have observed cover almost the same molecular weight range as those described by Oikawa $\mathrm{et}$ al. (1988); two-dimensional electrophoresis of follicular and ovulated zonae revealed two components with a similar molecular weight range of 150000-250000 (Fig. 2, a and b), one component of 90000 (c) and another of 55000 (d). Thus, (c) and (d) appear to be ZP2 and ZP3 respectively but the relationship between (a) and (b) with respect to ZP1 and a ZP0 is less clear. The fact that both follicular and ovulated zonae label at the higher molecular weight (a) does not rule out the possibility that the latter have acquired oviducal material, the molecular weights of ZPI and ZP0 overlapping as they do. Another possibility is that (a) and (b) are both components of the same molecule, ZP1, although their difference in distribution in the gel, i.e. (a) has signs of heterogeneity while (b) does not, and their quite different isoelectric points, argues against this. In terms of the compositional changes that appear in zonae from eggs fertilized in vivo our results might indicate that the disappearance of (b) has produced either the train (e) or (f) or both. Alternatively (e) could have pre-existed but with amino groups unavailable to label. The appearance of the low molecular weight polypeptide (f) on two dimensional gels but not one-dimensional gels is interesting, showing that after an initial cleavage the harsher dissociating conditions of the O'Farrell lysis buffer are required to separate this polypeptide from its precursor. However, without probes specific for individual zona (or oviducal) components it is not possible to interpret confidently the changes we see.

Noticeable from our two-dimensional gels is the fact that generally zona components did not separate into 'trains'. Since our electrophoretic technique clearly separate's subspecies (Fig. 2e) we considered it possible that in the hamster the method of zona preparation might influence the formation and resolution of subspecies. Oikawa et al. (1988), whose results did show zona components separating out into 'trains' (in the general manner), used hyaluronidase to remove cumulus oophorus and corona radiata from the zonae. As our zonae were denuded mechanically without enzymic assistance, and since crude hyaluronidase preparations may contain proteases, we repeated their approach by preparing zonae using 3 samples of bovine testicular hyaluronidase of differing purity (Sigma types I-S, IV-S and VI-S) and examined the resulting zonae by twodimensional electrophoresis. We saw no differences from results with the mechanically prepared zonae (results not shown). Another major difference in our method of zona preparation is that we labelled zonae immediately after they had been isolated in a balanced medium while Oikawa et al. (1988) stored zonae in distilled water at $-20^{\circ} \mathrm{C}$ (ovulated zonae) or freeze-dried (follicular zonae). However, such storage of zonae in other species appears to be without effect on zona composition as judged by two-dimensional electrophoresis.

Our one-dimensional gels appear to provide further evidence that the hamster zona pellucida takes up protein from the oviduct although our two-dimensional analyses do not emphasize this. An aspect of our experiments which might be obscuring the issue of uptake of oviduct proteins by the zona pellucida is the non-specificity of the label that we used. This non-specificity and the considerable overlap in molecular weights of hamster ZPI and ZP0 make it more difficult to distinguish between acquired oviducal protein and exposure of amino groups on existing zona proteins especially if the quantity of acquired protein is small.

Whilst we see a very significant increase of label incorporated into zonae fertilized in vivo our most striking observation remains that, in this situation, the investment undergoes considerable compositional modification, its profile becoming quite different from the other three categories examined. The question arises as to what causes the increase in amino groups and changes in composition in zonae fertilized in vivo. The fact that the oviducal environment alone appears to have no such effect on the zona of ovulated eggs, together with the observation that the zona of ovulated eggs fertilized in vitro is similarly unaffected, suggests that a synergism between oviducal and sperm factors may be operating on the zona. There seem to be several possibilities. It could be 
that, in vivo, damaged spermatozoa are releasing hydrolases into the oviduct, close to the egg; the confines of the oviduct may maintain effective concentrations whereas the considerable dilution factor encountered in the in-vitro fertilization procedure would be far less supportive. Alternatively, the presence of spermatozoa in the oviduct may trigger a response from the oviduct which yields factor(s) able to alter the zona in a manner not seen in vitro, but as far as we know there is no precedent for this. A further possibility is that proteases from cortical granules released at fertilization produce changes in the zona which are detectable after fertilization in vivo but which are not seen after the in-vitro system which is of much shorter duration. We tested this possibility by extending the in-vitro incubation to $10 \mathrm{~h}$ but, using two-dimensional analysis, we saw no difference in zona profile from the routine in-vitro fertilization procedure. However, it remains possible that a combined action of cortical secretory products and oviducal secretions could induce the changes we see. Another consideration is the effect that the supraphysiological levels of hCG administered for superovulation might be having on the chemistry of the oviduct and in turn on properties of the zona pellucida. Eggs fertilized in vivo were collected from animals that were not treated with hCG. It is conceivable that a high hCG concentration influences the oviduct to prevent superovulated unfertilized eggs from undergoing normal post-ovulatory changes.

Another question arises as to the biological significance, if any, of the modifications that occur in zonae from eggs fertilized in vivo; could products of such modifications become involved either in the sperm penetration process or in the nutrition, survival and development of the preimplantation embryo? For instance, in the mouse, glycoprotein $\left(M_{\mathrm{r}} 210000\right)$ from oviducal fluid passes across the zona pellucida to accumulate within the perivitelline space of the egg and embryo (Kapur $\&$ Johnson, 1986) and a similar phenomenon exists in the sheep (Gandolfi et al., 1989), which raises the possibility that these glycoproteins could bear elements for utilization by the developing embryo. However, this phenomenon may not be exclusive to oviducal macromolecules; polypeptides/peptides derived from alteration of the zona pellucida, generated either by the spermatozoa or by the oviduct, or both, might adopt essential roles in support of fertilization and early embryonic development in vivo.

Another consideration is that exposure of amino groups to the extent we see may modulate interactions between the early embryo and its immediate environment in the oviduct. For instance, the net charge of the embryo may become modified, as happens in the case of the erythrocyte when its surface-bound $\mathrm{N}$-acetylneuraminic acid is depleted by the enzyme neuraminidase (Cook et al., 1961; Eyler et al., 1962). Should this be the case it could have a bearing on such events as the migration of the early embryo through the oviduct into the uterus and shedding of the zona pellucida, processes about which virtually nothing is known at the molecular level.

We thank Dr Cesar Esper for his contribution to the supply of eggs fertilized in vitro; Dr D. Cran for advice and critical comments; Judy L. Williamson for skilled technical assistance; and Dianne Styles for typing the manuscript.

Supported in part by the National Institute of Child Health and Human Development, NIH, through cooperative agreement HD-22023 to B.D.B.

\section{References}

Ahuja, K.K. \& Bowell, G.P. (1983) Probable asymmetry in the organization of components of the hamster zona pellucida. J. Reprod. Fert. 69, $49-55$.

Bavister, B.D. (1989) A consistently successful procedure for in vitro fertilization of golden hamster eggs. Gamete Res. 23, 139-158.

Bavister, B.D. \& Yanagimachi, R. (1977) The effects of sperm extracts and energy sources on the motility and acrosome reaction of hamster spermatozoa in vitro. Biol. Reprod. 16, 228-237.
Brown, C.R. \& Cheng, W.K.T. (1985) Limited proteolysis of the porcine zona pellucida by homologous sperm acrosin. J. Reprod. Fert. 74, 257-260.

Brown, C.R. \& Cheng, W.K.T. (1986) Changes in composition of the porcine zona pellucida during development of the oocyte to the 2-to-4-cell embryo. $J$. Embryol. exp. Morph. 92, 183-191.

Boatman, D.E. (1990) Oviductal modulators of sperm fertilizing ability. In Fertilization in Mammals (in press). Eds B. D. Bavister, J. M. Cummins \& E. Roldan. Serono, Norwell. 
Bolton, A.E. \& Hunter, W.M. (1973) The labelling of proteins to high specific activities by conjugation to a ${ }^{125}$ I-containing acylating agent. Biochem. J. 133, 529-539.

Cook, G.M.W., Heard, D.H. \& Seaman, G.V.F. (1961) Sialic acids and the electrokinetic charge of the human erythrocyte. Nature, Lond. 191,44-47.

Dietl, J. (Ed.) (1988) The Mammalian Egg Coat: Structure and Function. Springer Verlag, Berlin.

Eyler, E.H., Madoff, M.A., Brody, O.V. \& Oncley, J.L. (1962) The contribution of sialic acid to the surface charge of the erythrocyte. J. biol. Chem. 237, 19922000.

Gandolfi, F., Brevini, T.A.L., Richardson, L., Brown, C.R. \& Moor, R.M. (1989) Characterization of proteins secreted by sheep oviduct epithelial cells and their function in embryonic development. Development 106, 303-312.

Gwatkin, R.B.L. (1978) Cell-cell interaction in mammatian fertilization. In The Molecular Basis of CellCell interaction, pp. 363-376. Eds R. A. Lerner \& D. Bergsma. A. R. Liss, New York.

Kapur, R.P. \& Johnson, L.V. (1986) Selective sequestration of an oviductal fluid glycoprotein in the perivitelline space of mouse oocytes and embryos. J. exp. Zool. 238, 249-260.

Lévéillé, M.C., Roberts, K.D., Chevalier, S., Chapdelaine, A. \& Bleau, G. (1987) Uptake of an oviductal antigen by the hamster zona pellucida. Biol. Reprod. 36, 227-238.

O'Farrell, P.H. (1975) High resolution two-dimensional electrophoresis of proteins. J. biol. Chem. 250, 40074021.

Oikawa, T., Sendai, Y., Kuratam, S. \& Yanagimachi, R. (1988) A glycoprotein of oviductal origin alters biochemical properties of the zona pellucida of hamster egg. Gamete Res. 19, 113-122.

Sacco, A.G., Subramanian, M.G. \& Yurewicz, E.C. (1984) Association of sperm receptor activity with a purified pig zona antigen (PPZA). J. Reprod. Immunol. 6, 89-103.

Sacco, A.G., Yurewicz, E.C., Subramanian, M.G. \& Matzat, P.D. (1989) Porcine zona pellucida: association of sperm receptor activity with the $\alpha$-glycoprotein component of the $\mathrm{M}_{\mathrm{r}}=55000$ family. Biol. Reprod. 41, 523-532.

Wassarman, P., Bleil, J., Fimiami, C., Florman, H., Greve, J., Kinlock, R., Moller, C., Montillo, S., Roller, R., Saltzman, G. \& Vazquez, M. (1988) The mouse egg receptor for sperm: a multifunctional zona pellucida glycoprotein. In The Mammalian Egg Coat: Structure and Function, pp. 18-37. Ed. J. Dietl Springer Verlag, Berlin.

Received 2 February 1990 